\section{Change in guardians' preference for computed tomography after explanation by emergency physicians in pediatric head injury}

Jin Hee Jeong ${ }^{1}$, Jin Hee Lee ${ }^{2,3}$, Kyuseok Kim ${ }^{4}$ Joong Eui Rhee ${ }^{5}$, Tae Yun Kim ${ }^{4}$, You Hwan Jo ${ }^{4}$, Yu Jin Kimª , Jae Hyuk Lee ${ }^{4}$, Changwoo Kang' ${ }^{1}$, Soo Hoon Lee', Joonghee Kim ${ }^{4}$, Chan Jong Park ${ }^{6}$, Hyuksool Kwon ${ }^{4}$

Department of Emergency Medicine, ${ }^{1}$ Gyeongsang National University Hospital, Jinju; ${ }^{2}$ Seoul National University Hospital, Seoul; ${ }^{3}$ Kangwon National University School of Medicine, Chuncheon; ${ }^{4}$ Seoul National University Bundang Hospital, Seongnam; ${ }^{5}$ Samsung Medical Center, Seoul; ${ }^{6}$ Veterans Health Service Medical Center, Seoul, Korea

Objective Head injury in children is a common problem presenting to emergency departments, and cranial computed tomography scanning is the diagnostic standard for these patients. Several decision rules are used to determine whether computed tomography scans should be used; however, the use of computed tomography scans is often influenced by guardians' preference toward the scans. The objective of this study was to identify changes in guardian preference for minor head injuries after receiving an explanation based on the institutional clinical practice guideline.

Methods A survey was conducted between July 2010 and June 2012. Patients younger than 16 years with a Glasgow Coma Scale score of 15 after a head injury and their guardians were included. Pre- and post-explanation questionnaires were given to guardians to assess their preference for computed tomography scans and factors related to the degree of preference. Treating physicians explained the risks and benefits of cranial computed tomography scanning using the institutional clinical practice guideline. Guardian preference for a computed tomography scan was examined using a 100-mm visual analog scale.

Results In total, 208 patients and their guardians were included in this survey. Guardian preference for computed tomography scans was significantly reduced after explanation (46.7 vs. 17.4, $P<0.01)$. Pre-explanation preference and the strength of the physician recommendation to get a computed tomography were the most important factors affecting pre- and post-explanation changes in preferences.

Conclusion Explanation of the risks and benefits of cranial computed tomography scans using the institutional clinical practice guideline may significantly reduce guardian preference for computed tomography scans.

Keywords Head injuries, closed; Pediatrics; Tomography, X-ray computed
elSSN: 2383-4625

Received: 15 June 2015

Revised: 11 September 2015

Accepted: 12 September 2015

Correspondence to: Jin Hee Lee Department of Emergency Medicine, Seoul National University Hospital, 101 Daehak-ro, Jongno-gu, Seoul 03080, Korea

E-mail: gienee@snubh.org

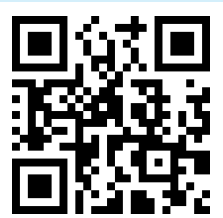

How to cite this article:

Jeong JH, Lee JH, Kim K, Rhee JE, Kim TY, Jo YH, Kim YJ, Lee JH, Kang C, Lee SH, Kim J, Park CJ, Kwon H. Change in guardians' preference for computed tomography after explanation by emergency physicians in pediatric head injury. Clin Exp Emerg Med 2015;2(4):226-235.
This is an Open Access article distributed under the terms of the Creative Commons Attribution Non-Commercial License (http:// creativecommons.org/licenses/by-nc/3.0/). 


Capsule
$\begin{aligned} & \text { What is already known } \\ & \text { Cranial computed tomography scan is the diagnostic standard for intracranial injury. However, the radiation risk of } \\ & \text { computed tomography scans is problematic, particularly in children. }\end{aligned}$
$\begin{aligned} & \text { What is new in the current study } \\ & \text { When physicians explain the risks and benefits of cranial computed tomography scans using the institutional clinical } \\ & \text { practice guideline, guardian preference for computed tomography scans can be reduced. }\end{aligned}$

\section{INTRODUCTION}

Head injury in children is a common problem presenting to emergency departments (EDs). ${ }^{1,2}$ Cranial computed tomography (CT) scans are the diagnostic standard for identifying the presence of intracranial injury in the acute phase. ${ }^{3}$ However, the radiation risk of CT scans should be considered, particularly in children. ${ }^{4}$ Decisions about CT scans are challenging for minor head injuries, because it is difficult to balance radiation risk and identification of intracranial injuries. ${ }^{1,2}$ Several decision rules have been developed to help with decision-making for CT scans, but these were based on limited populations. ${ }^{3,5-7}$ In 2009, the Pediatric Emergency Care Applied Research Network (PECARN) rule was derived and validated for children with minor head injuries using Glasgow Coma Scale (GCS) scores of 14 to 15 in a large population of children. ${ }^{8}$ This rule was developed to identify clinically important traumatic brain injuries (CiTBI) and reduce excessive CT use. ${ }^{8}$ We developed and applied an institutional clinical practice guideline based on published decision rules to reduce $\mathrm{CT}$ use. ${ }^{5-8}$ During application of this practice guideline, CT scan use was also influenced by guardian preference. We hypothesized that an explanation based on the practice guideline could change guardian preference. Therefore, the objective of this study was to identify changes in guardian preferences after an explanation of the risks and benefits of cranial CT scans using a clinical practice guideline.

\section{METHODS}

\section{Study design and setting}

This study was a survey including pre- and post-explanation questionnaires (Appendices 1-3). The methodology was approved by the hospital institutional review board. The setting was the ED of a tertiary hospital with an annual census of 26,000 pediatric patients (younger than 16 years). Patients were treated by emergency resident physicians supervised by board-certified attending physicians.

Our institutional clinical practice guideline for pediatric minor head injury was developed by an institutional joint committee of EP and pediatric neurologists. ${ }^{6-8}$ The committee reviewed several published decision rules including the PECARN rule, ${ }^{6-8}$ and modified these rules for our institution. The clinical practice guideline could be applied to children with blunt minor head injuries with GCS greater than 13. Cranial CT scans were used to identify any patients with a CiTBI, defined as death from head injury, neurosurgery, intubation for more than 24 hours, or hospital admission for at least two nights associated with head injuries on CT scans. ${ }^{8}$

\section{Survey study protocol}

The clinical practice guideline was implemented beginning in July 2010. During 6 months prior to the survey, we educated emergency physicians (EP) about the new guideline and posted it for them to consult at any time. The survey was conducted between July 2010 and June 2012 from 10 am to $10 \mathrm{pm}$. The study period was limited due to the working time of clinical research nurses. Inclusion criteria were age younger than 16 years, GCS of 15 , and treatment in our ED after head injuries. Patients with preexisting neurologic diseases or coagulation diseases, those with non-blunt head injuries or with injuries to other body parts, and those who were transported after head imaging studies at other hospitals were excluded from the survey. When an eligible patient arrived at the ED, the triage nurse gave the guardian the preliminary questionnaire form after describing the study. After the guardian completed the questionnaire, a board-certified EP examined the patient and explained the risks and benefits of CT scans, and symptoms and signs that required a CT scan, based on the clinical practice guideline. After hearing the explanation, the guardian filled out the post-explanation questionnaire. Informed consent of guardians was also obtained. The preliminary questionnaire asked about patient characteristics such as sex, age, presence of siblings, experience, numbers of previous CT scans, injury time, mechanism of injury, and symptoms related to the injury. The symptoms related to the injury included amnesia, loss of consciousness, seizure, irritability, headache, dizziness, vomiting, 
lethargy, and abnormal behavior. We also collected data about the guardian, including sex, age, relationship to the patient, education level, severity of the injury as perceived, and guardian preference toward a CT scan. It was not possible to assess whether patients younger than 3 years had amnesia, headache, or dizziness. The post-explanation questionnaire asked about previous knowledge about the risk of CT scans, how they learned about the risk, the degree to which the physician recommended CT, and guardian preference toward a CT scan. The severity of the injury as perceived by guardians, the degree to which the physician recommended $\mathrm{CT}$, and guardian preference toward a CT scan were assessed in the pre- and post-explanation questionnaires using a $100-\mathrm{mm}$ visual analog scale. A value of "0" indicated that the guardian did not want the use of CT scans.

Ninety days after discharge, a follow-up telephone call was made to identify symptoms and management in other hospitals due to head injuries. ${ }^{8}$ We assessed changes in guardian preference between pre- and post-explanation questionnaires (preand post-explanation preference) and factors related to changes in preferences.

Table 1. Baseline characteristics of survey patients

\begin{tabular}{|c|c|}
\hline Clinical variable & Patient $(n=208)$ \\
\hline \multicolumn{2}{|l|}{ Sex } \\
\hline Male & $128(61.5)$ \\
\hline Female & $80(38.5)$ \\
\hline Age (yr) & $3.6 \pm 3.3$ \\
\hline Presence of sibling & $107(51.4)$ \\
\hline Previous experience with CT scans & $20(9.6)$ \\
\hline Previous number of CT scans & $1.2 \pm 0.5$ \\
\hline \multicolumn{2}{|l|}{ Mechanism of injury } \\
\hline Falling & $99(47.6)$ \\
\hline Collision & $56(26.9)$ \\
\hline Slipping down & $53(25.5)$ \\
\hline \multicolumn{2}{|l|}{ Symptoms of patients } \\
\hline Amnesiaa) & $5(4.7)$ \\
\hline Loss of consciousness ${ }^{a)}$ & $9(4.3)$ \\
\hline Seizure & $1(0.5)$ \\
\hline Irritability & $51(24.5)$ \\
\hline Headache $^{\text {a) }}$ & $82(77.4)$ \\
\hline Dizziness & $36(34.0)$ \\
\hline Vomiting & $48(23.1)$ \\
\hline Lethargy & $45(21.6)$ \\
\hline Abnormal behavior & $43(20.7)$ \\
\hline \multicolumn{2}{|l|}{ Signs of patients } \\
\hline Focal neurologic deficit & $0(0)$ \\
\hline Scalp hematoma & $63(30.3)$ \\
\hline Scalp laceration & $3(1.4)$ \\
\hline Scalp fracture sign & $0(0)$ \\
\hline Bulging fontanel & $0(0)$ \\
\hline Performance of skull radiograph & 79 (38.0) \\
\hline
\end{tabular}

Values are presented as number (\%) or mean \pm standard deviation.

$\mathrm{CT}$, computed tomography.

a)These findings were sought in 106 patients.

\section{Outcomes}

The primary outcomes were changes in guardian preferences after receiving an explanation according to the new clinical practice guideline. The secondary outcomes were the related factors affecting changes in guardian preferences.

\section{Data analysis}

Statistical analysis was performed using the IBM SPSS Statistics ver. 19.0 (Armonk, NY, IBM Corp., USA). In the analysis of the survey study, pre- and post-explanation guardian preference was compared using the paired t-test. Factors affecting these changes were identified using univariate linear regression, and factors with P-values less than 0.1 were included in multivariate linear regression. Correlations between knowledge of the risk of CT scans, experience with previous CT scans, highest education level, and pre-explanation preference were identified using the $X^{2}$ test and Student's t-test. A P-value less than 0.05 was considered statistically significant.

\section{RESULTS}

A total of 208 patients were surveyed during the study period. Male patients accounted for 61.5\% (128 patients) and the mean age was 3.6 (standard deviation 3.3) years (range, 2 months to 15

Table 2. Baseline characteristics of guardians

\begin{tabular}{lc}
\hline Clinical variable & Guardian $(\mathrm{n}=208)$ \\
\hline Sex & \\
Male & $46(22.1)$ \\
Female & $156(75.0)$ \\
Age (yr) & $36.8 \pm 4.5$ \\
Relationship with patients & \\
Parents & $200(96.2)$ \\
Teacher & $1(0.5)$ \\
Highest level of education & \\
High school graduate & $11(5.3)$ \\
In college or graduated & $151(72.6)$ \\
Graduate school & $40(19.2)$ \\
Knowledge about radiation risk & $98(47.1)$ \\
Source of knowledge about radiation & \\
The mass media & $50(24.0)$ \\
Hospitals & $26(12.5)$ \\
Acquaintances & $16(7.7)$ \\
Internet & $9(4.3)$ \\
Other & $6(2.9)$ \\
Pre-questionnaire preference of guardians & $46.7 \pm 32.0$ \\
Post-questionnaire preference of guardians & $17.4 \pm 19.3$ \\
Strength of physicians recommended CT & $17.6 \pm 17.6$ \\
Severity of injury perceived by guardians & $27.6 \pm 17.7$ \\
\end{tabular}

Values are presented as number (\%) or mean \pm standard deviation. There were some missing values.

$\mathrm{CT}$, computed tomography. 
Jin Hee Jeong, et al.

Table 3. Factors affecting change in preference between pre- and post-explanation questionnaires

\begin{tabular}{|c|c|c|c|c|}
\hline \multirow{2}{*}{ Variable } & \multirow{2}{*}{$\beta$ (SE) } & \multirow{2}{*}{ P-value } & \multicolumn{2}{|c|}{$95 \% \mathrm{Cl}$} \\
\hline & & & Lower & Upper \\
\hline Sex of patients & $2.21(4.41)$ & 0.62 & -6.48 & 10.90 \\
\hline Age of patients & $-0.33(0.65)$ & 0.61 & -1.61 & 0.95 \\
\hline Presence of sibling & $6.48(4.35)$ & 0.14 & -2.10 & 15.06 \\
\hline Previous experience with CT scans & $-2.54(7.26)$ & 0.73 & -16.86 & 11.77 \\
\hline Body part of CT scans in previous experiences & 7.85 (9.29) & 0.41 & -11.96 & 27.66 \\
\hline Mechanism of injury & $-1.97(2.56)$ & 0.47 & -6.91 & 3.18 \\
\hline Performance of skull radiograph & $3.05(4.40)$ & 0.49 & -5.63 & 11.73 \\
\hline Sex of guardians & $-2.44(5.14)$ & 0.64 & -12.57 & 7.70 \\
\hline Age of guardians & $-0.54(0.51)$ & 0.30 & -1.55 & 0.47 \\
\hline Relationship with patients & $-16.52(14.82)$ & 0.27 & -45.75 & 12.72 \\
\hline Highest level of education of guardians & $-1.19(4.74)$ & 0.80 & -10.54 & 8.17 \\
\hline Knowledge about radiation risk of guardians & $7.60(4.34)$ & 0.08 & -0.95 & 16.15 \\
\hline Source of knowledge about radiation & $1.21(1.99)$ & 0.55 & -2.74 & 5.15 \\
\hline Pre-questionnaire preference of guardians & $0.78(0.04)$ & $<0.01$ & 0.70 & 0.85 \\
\hline Strength of physician recommended CT & $-0.25(0.12)$ & 0.04 & -0.49 & -0.01 \\
\hline Severity of injury as perceived by guardians & $0.20(0.12)$ & 0.10 & -0.04 & 0.44 \\
\hline
\end{tabular}

$\mathrm{SE}$, standard error; $\mathrm{Cl}$, confidence interval; $\mathrm{CT}$, computed tomography.

Table 4. Factors affecting change in preference between pre- and post-explanation questionnaires, based on multiple linear regression analysis

\begin{tabular}{lrrrr}
\hline Variable & & & \multicolumn{2}{c}{$95 \% \mathrm{Cl}$} \\
\cline { 3 - 4 } & & P-value & Lower & Upper \\
\hline Knowledge about radiation risk of guardians & $2.40(2.09)$ & 0.25 & -1.72 \\
Pre-questionnaire preference of guardians & $0.85(0.03)$ & $<0.01$ & 6.52 \\
Strength of physician recommended CT & $-0.59(0.06)$ & $<0.01$ & 0.78 \\
\hline
\end{tabular}

$\mathrm{SE}$, standard error; $\mathrm{Cl}$, confidence interval; $\mathrm{CT}$, computed tomography.

years). Twenty patients (9.6\%) had undergone CT scans previously. The most common cause of injury was a fall. Headache was the most common symptom, and scalp hematoma was the most common sign. No patients had a focal neurologic deficit, sign of a scalp fracture, or bulging fontanel. Although skull radiographs were performed in 78 patients, no fractures were detected (Table 1).

Female guardians accounted for $75.0 \%$, and most guardians were parents. About half of the guardians knew about the radiation risk of $\mathrm{CT}$ scans, and half had obtained this information from the mass media (Table 2). Knowledge about the radiation risk of CT scans was not associated with previous experience with $\mathrm{CT}_{\text {, }}$ highest level of education, or pre-explanation preference.

Guardian preference for $\mathrm{CT}$ scans was significantly reduced after receiving an explanation (46.7 vs. $17.4 ; \mathrm{P}<0.01 ; 95 \%$ confidence interval, 25.1 to 33.5). Change in preference before and after explanation, as measured using the VAS, was significantly affected by pre-explanation preference and the strength of the physician recommended CT. However, change in preference was not significantly associated with age, presence of siblings, previous experiences with CT scans, mechanism of injury, symptoms, signs, or performance of skull radiography. Variables related to guardians, such as sex, age, relationship to the patient, highest education level, knowledge about radiation risk, source of knowledge about radiation, and severity of the injury as perceived by guardians, were not significantly related to change in preferences (Table 3). Multivariate analysis revealed that the most important factors affecting changes in preference were pre-explanation preference and the strength of the physicians recommendation for a CT (Table 4).

At 90 days, three patients were not assessed for their symptoms. The remaining patients had recovered from their symptoms and were not being managed in other hospitals. The proportion of children who received CT scans after implementing the practice guideline was significantly reduced compared with that before the practice guideline period ( $7.3 \%$ vs. $13.6 \%, \mathrm{P}<0.01)$.

\section{DISCUSSION}

This is the first study to explore changes in guardian preferences for CT scans after receiving an explanation by physicians about 
the risks and benefits of the clinical practice guideline. Guardian preference was significantly reduced after explanation, and this change was associated with pre-explanation preference and the strength of the physicians recommended CT.

Since the introduction of CT scans in the 1970s, their use has increased among both adults and children. ${ }^{4}$ The use of head CT scans for minor head injuries has doubled over 10 years. ${ }^{2} \mathrm{CT}$ scans have a radiation risk, and children are at more risk from radiation than adults because of radiosensitive tissue and longer life duration. ${ }^{9}$ Radiation exposure from CT scans may also increase the risk of leukemia and brain tumors in children. ${ }^{10}$ The estimated rate of cancer in children after head CT scans is reportedly between 1 in 1,000 and 1 in 5,000, ${ }^{8,11}$ However, approximately half of the guardians in our survey study did not know about the radiation risk of CT scans. One previous study reported that guardian knowledge of radiation risk associated with CT scans was not affected by previous use of CT scans among their children. ${ }^{12}$ These authors emphasized education about radiation for guardians. Our results also indicate that there is no relationship between knowledge about radiation risk and previous use of CT. This might mean that the knowledge that guardians had was abstract and unreasonable; moreover, they were unaware of when children required CT scans. Therefore, explanation of the radiation risk using the evidence-based decision rule, which clarified which cases required CT scans, could significantly reduce preferences. Two factors were significantly related to change in preference: pre-explanation preference and the strength of the physician's recommendation for the CT.

Pre-explanation preference was not associated with knowledge about radiation risk. This suggests that greater pre-explanation preference would result in a greater change in preference, i.e., preference was significantly reduced by explanation.

Physicians have difficulty deciding whether CT scans are required for minor head injuries due to the variability in management, $^{5,11}$ and should make careful decisions about the use of CT scans, considering the risks and benefits to children. EPs at our hospital have also had difficulty with this decision. An institutional clinical practice guideline may help EPs make decisions as well as help them to explain these decision to guardians more reasonably. Because the strength of the physician's recommendation for a CT could differ (based on the degree of necessity of CT scans according to the guideline), this might affect post-explanation preference and change in preference. Among the factors identified as affecting a change in preference, the strength of the physician's recommendation for a CT was the only adjustable factor in the ED. This might be used to reduce the number of unnecessary CT scans that are requested by guardians.
This study had several limitations. First, this was a survey study that included some, but not all, children with head injuries. Therefore, the results may reveal reduced preference among their guardians. Because we could not access the characteristics of the unenrolled patients, we identified the performance rate of CT scans after the guideline period. We assumed that the clinical practice guideline affected the use of CT scans based on the lower numbers of CT scans performed after guideline period. Second, because the survey was conducted from $10 \mathrm{am}$ to $10 \mathrm{pm}$, selection bias may have been involved. The limited study time may also have had an effect in that all suspected patients were not included. However, the characteristics of guardians and patients may not have been different during other time periods. Third, as the hospital is located in a metropolitan area, the patients and guardians visiting this hospital represent a reasonably well-educated sample; thus, they may better understand the explanation of physicians. Therefore, the results may not necessarily apply to a less educated population. Fourth, this study was conducted in a single center, so the ability to generalize the results is limited.

In conclusion, explanation of the risks and benefits of cranial CT scans using the clinical practice guideline may significantly reduce guardian preference toward CT.

\section{CONFLICT OF INTEREST}

No potential conflict of interest relevant to this article was reported.

\section{ACKNOWLEDGMENTS}

This study was supported by grant number 11-2010-025 from Seoul National University Bundang Hospital research fund.

\section{REFERENCES}

1. Tavarez MM, Atabaki SM, Teach SJ. Acute evaluation of pediatric patients with minor traumatic brain injury. Curr Opin Pediatr 2012;24:307-13.

2. Hennelly KE, Mannix R, Nigrovic LE, et al. Pediatric traumatic brain injury and radiation risks: a clinical decision analysis. J Pediatr 2013;162:392-7.

3. Palchak MJ, Holmes JF, Vance CW, et al. A decision rule for identifying children at low risk for brain injuries after blunt head trauma. Ann Emerg Med 2003;42:492-506.

4. Brenner DJ, Hall EJ. Computed tomography: an increasing source of radiation exposure. N Engl J Med 2007;357:227784. 
5. Sultan HY, Boyle A, Pereira M, Antoun N, Maimaris C. Application of the Canadian $\mathrm{CT}$ head rules in managing minor head injuries in a UK emergency department: implications for the implementation of the NICE guidelines. Emerg Med J 2004; 21:420-5.

6. Dunning J, Daly JP, Lomas JP, et al. Derivation of the children's head injury algorithm for the prediction of important clinical events decision rule for head injury in children. Arch Dis Child 2006;91:885-91.

7. Osmond MH, Klassen TP, Wells GA, et al. CATCH: a clinical decision rule for the use of computed tomography in children with minor head injury. CMAJ 2010;182:341-8.

8. Kuppermann N, Holmes JF, Dayan PS, et al. Identification of children at very low risk of clinically-important brain injuries after head trauma: a prospective cohort study. Lancet 2009;
374:1160-70.

9. Nigrovic LE, Schunk JE, Foerster $A$, et al. The effect of observation on cranial computed tomography utilization for children after blunt head trauma. Pediatrics 2011;127:1067-73.

10. Pearce MS, Salotti JA, Little MP, et al. Radiation exposure from CT scans in childhood and subsequent risk of leukaemia and brain tumours: a retrospective cohort study. Lancet 2012;380: 499-505.

11. Fundaro $C$, Caldarelli $M$, Monaco $S$, et al. Brain $C T$ scan for pediatric minor accidental head injury: an Italian experience and review of literature. Childs Nerv Syst 2012;28:1063-8.

12. Hartwig HD, Clingenpeel J, Perkins AM, Rose W, Abdullah-Anyiwo J. Parental knowledge of radiation exposure in medical imaging used in the pediatric emergency department. Pediatr Emerg Care 2013;29:705-9. 
Appendix 1. Preliminary questionnaires

1. State the time of the child's injury.

Year: Month: Date: Hour: Minute:

2. Describe how the injury happened.

3. On a scale of 0 to 10 , rate how severe the injury seems to you. Score 0 indicates that you consider the injury to have little or no significance for the child's health whereas score 10 means that "you regard the injury as life-threatening."

It seems that there is no problem associated with the injury.
It seems that the head injury is

life threatening head injury.

4. Please check all of the symptoms of the child.

$\square$ Amnesia (the child was unable to recall the accident)

$\square$ Loss of consciousness for minutes

Seizure, convulsion

$\square$ Irritability

$\square$ Headache

Dizziness

Number of vomiting episodes:

Lethargy

$\square$ Change in the child's behavior after injury?

5. On the scale of 0 to 10 , indicate how much you were willing to have the child undergo a brain computed tomography (CT).

I am strongly against the idea of my child undergoing a brain CT.
I strongly want my child to undergo a brain CT. 
Appendix 2. Explanation for patients of minor head injury

Definition of minor head injury

Minor head injury is a case in which a patient with head injury shows minimal symptoms and signs, and no clinically important traumatic brain injury is observed via either physical examination or brain computed tomography (CT). Reliable studies indicate that if there are no symptoms or abnormalities found in the physical examination, clinically important traumatic brain injuries are not present in 99\% of patients. In such a case, the patient is discharged without undergoing a CT scan from the hospital and is observed at home for development of any symptoms.

Watch carefully for the following symptoms and signs.

1) Any change of mental status: abnormal behaviors, lethargy, change in sleeping tendency, greatly increased difficulty in waking the child

2) Prolonged nausea or vomiting more than 5 times

3) Headache does not improve or gets worse

4) Continued dizziness or difficulty in balancing

5) Sensory changes or abnormal movement in the extremities

6) Visual disturbance

Explanation of CT scans

Infants and children may experience several incidents of vomiting after a minor impact on the head even in the absence of clinically important traumatic brain injury. Hence, guardians do not need to worry too much even though the patient has vomited a few times.

1) However, guardians should notice whether vomiting gets worse, the child's mental status alters, or headache persists.

2) Refrain from obtaining a radiological examination if possible. When infants undergo a CT scan, they are exposed to more than 1,000 times the radiation of a chest X-ray. Children are more vulnerable to the risk of radiation from CT scanning. If the infant is exposed to one brain CT scan, the cancer-related death risk is 1 to 8 per 10,000 persons. Therefore, the decision of undergoing a radiology examination should be made after comparing the risks and benefits. 
Appendix 3. Post-explanation questionnaires

1. Now, having listened to an explanation regarding computed tomography (CT) scans, on a scale of 0 to 10 , indicate how much you were willing to have the child undergo a brain CT.

I was strongly against the idea of my child undergoing a brain CT.
I strongly wanted my child to undergo a brain CT.

2. Indicate how strongly the doctor recommended CT on the scale of 0 to 10.

CT scanning is not necessary at all.

CT scans is absolutely necessary.

\section{Baseline characteristics}

1) How many brothers or sisters does the injured child have?

Number of brothers:

Number of sisters:

Birth order of the child: 1 st/2nd/3rd/ th

2) What is the highest education level of the injured child's guardian who made the decision whether to undergo a CT scan?

$\square$ Elementary school graduate

$\square$ Middle school graduate

$\square$ High school graduate

$\square$ In college or graduated

$\square$ Graduate school

$\square$ Others:

3) Did you know that CT scans increase the possibility of cancer incidence?

$\square$ Yes, I did.

$\square$ No, I did not.

4) (Skip this question if you answered 'No' in question 3) Where did you learn that CT scans increase the risk of cancer?

$\square$ From mass media

$\square$ From a hospital

$\square$ From an acquaintance/friend

$\square$ Over the internet

$\square$ Other: 
Jin Hee Jeong, et al.

Has the injured child previously undergone a CT scan? If so, 5) how many times has the child undergone a CT scan?

$\square$ Yes time(s)

$\square$ No

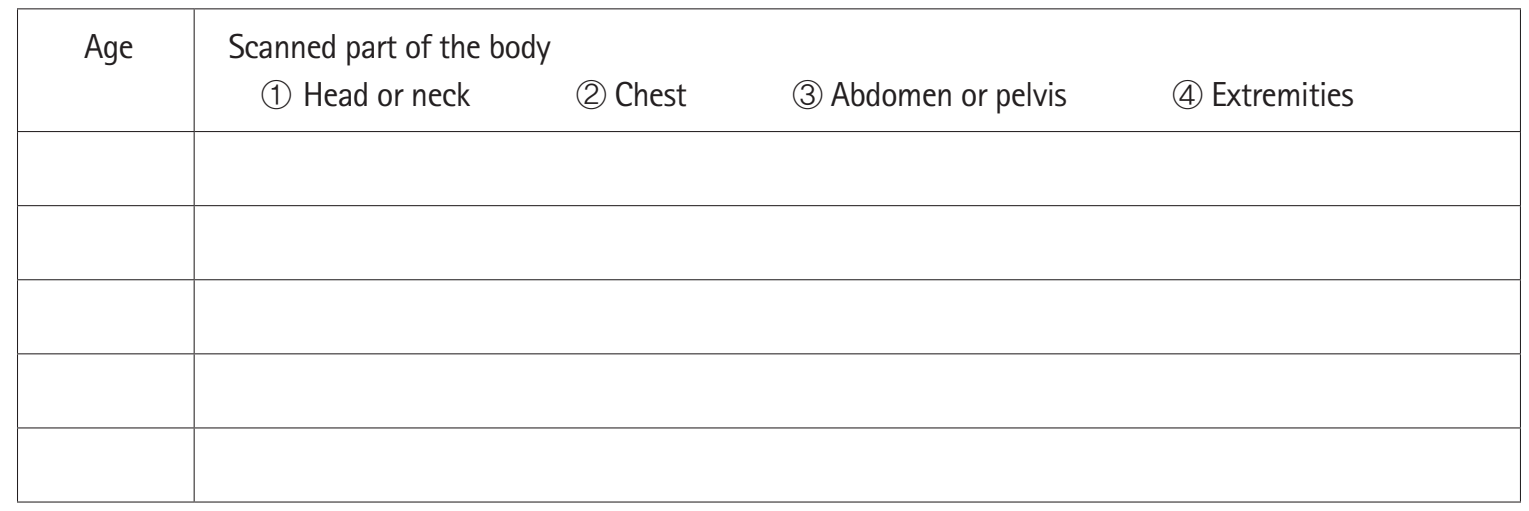

